\title{
"Una os alimentos" - brincando com os alimentos a partir de vivência em sala de aula
}

"Unite the foods" - playing with food through classroom experience

"Une los alimentos" - jugar con los alimentos a partir de la experiencia en el aula

Michele Aparecida Silva Maciel

ORCID: https://orcid.org/0000-0002-0733-0876

Universidade Federal de Uberlândia, Brasil

E-mail: michele_unitri@hotmail.com

Mariana Ramos

ORCID: https://orcid.org/0000-0003-3230-8424

Universidade Federal de Uberlândia, Brasil

E-mail: mari.ramos2009@hotmail.com

Cecília Soares Ferreira Carilli

ORCID: https://orcid.org/0000-0002-6742-8883

Universidade Federal de Uberlândia, Brasil E-mail:carillicecilia.cc@gmail.com

Ludmylla Soares Carrijo

ORCID: https://orcid.org/0000-0002-3471-1201

Universidade Federal de Uberlândia, Brasil

E-mail: ludmyllacarrijo@gmail.com

Isabela Caroline Gonçalves Simões

ORCID: https://orcid.org/0000-0003-4649-9583

Universidade Federal de Uberlândia, Brasil E-mail: isasimoes34@gmail.com

Mariane Bruna da Silva Mendes

ORCID: https://orcid.org/0000-0002-7529-3720

Universidade Federal de Uberlândia, Brasil

E-mail:mariane.bruna@hotmail.com

Leonardo Daniel Reis Santos

ORCID: https://orcid.org/0000-0002-2774-9842

Universidade Federal de Uberlândia, Brasil

E-mail:leoanardoudi2016@gmail.com

Patrícia Costa dos Santos da Silva

ORCID: https://orcid.org/0000-0001-9643-1865

Universidade Federal de Uberlândia, Brasil E-mail: patriciacosta@ufu.br

Andrea Mara Bernardes da Silva

ORCID: https://orcid.org/0000-0001-5126-1110

Universidade Federal de Uberlândia, Brasil

E-mail: andrea-bernardes@hotmail.com

Luana Araújo Macedo Scalia

ORCID: https://orcid.org/0000-0003-1000-8738

Universidade Federal de Uberlândia, Brasil

E-mail: luanascalia@ufu.br

\begin{abstract}
Resumo
Objetivo: Descrever a experiência, desenvolvida através da metodologia ativa em sala de aula, na criação de um jogo como forma de ensinar sobre alimentação saudável para crianças de seis a onze anos através do lúdico. Metodologia: O presente artigo trata-se de um relato de experiência descritivo, reflexivo, criado como proposta da disciplina de Assistência Integral à Saúde da Criança e do Adolescente I do curso de graduação em Enfermagem da Universidade Federal de Uberlândia, de maio a junho de 2019. O jogo foi aplicado em sala com 30 estudantes da graduação em enfermagem, subdivididos em 3 grupos de 10 pessoas. Para condução da brincadeira os autores do trabalho se distribuíram nos grupos menores como moderadores, assim foi possível observar a interação dos participantes das atividades. Ao final, os envolvidos apresentaram feedback positivo da ação. Discussão: O jogo foi planejado com base no o saber anterior da criança e o ponto de partida do seu desenvolvimento e buscou explorar de forma lúdica o âmbito sócio cognitivo. Para os acadêmicos, permitiu vivenciar as dificuldades no desenvolvimento de recursos didáticos e ao mesmo tempo evidenciou a importância do lúdico no ensino de crianças. Considerações finais: A atividade proposta foi fundamental para proporcionar reflexões de como atividades lúdicas são importantes para o aprendizado das crianças. O enfermeiro possui o papel de educador e através de jogos deve auxiliar as crianças a se cuidarem e promover saúde.
\end{abstract}

Palavras-chave: Saúde da criança; Nutrição da Criança; Jogos e Brinquedos; Ensino. 


\begin{abstract}
Objective: To describe the experience, developed through active methodology in the classroom, of creating a game as a method of teaching about healthy eating for children aged six to eleven years old through playfulness. Methodology: This article is a descriptive, reflective experience report, created as a proposal of the discipline of Integral Assistance to Child and Adolescent Health I of the undergraduate course in Nursing at the Federal University of Uberlândia, from May to June 2019. The game was applied in class with 30 undergraduate nursing students, subdivided into 3 groups of 10 people. To conduct the game, the authors of the paper distributed themselves in the smaller groups as moderators, so it was possible to observe the interaction of the participants of the activities. Discussion: The game was planned based on the previous knowledge of the child and the starting point of their development and sought to explore in a playful way the social-cognitive sphere. For the students, it allowed them to experience the difficulties in developing teaching resources and at the same time highlighted the importance of play in teaching children. Final considerations: The proposed activity was fundamental to provide reflections on how important playful activities are for children's learning. The nurse has the role of an educator, and through games should help children to take care of themselves and promote health.
\end{abstract}

Keywords: Child Health; Child Nutrition; Games and Toys; Teaching.

\title{
Resumen
}

Objetivo: Describir la experiencia, desarrollada a través de la metodología del aula activa, de crear un juego como forma de enseñar sobre alimentación saludable a niños de seis a once años a través del juego. Metodología: Este artículo es un informe de experiencia descriptivo y reflexivo, creado como propuesta de la disciplina Cuidados Integrales para la Salud del Niño y del Adolescente I del curso de pregrado en Enfermería de la Universidad Federal de Uberlândia, de mayo a junio de 2019. El juego se aplicó en el aula con 30 estudiantes de enfermería, subdivididos en tres grupos de 10 personas. Para llevar a cabo el juego, los autores del estudio se distribuyeron en los grupos más pequeños como moderadores, por lo que fue posible observar la interacción de los participantes en las actividades. Al final, los involucrados presentaron una retroalimentación positiva de la acción.Discusión: El juego fue planeado con base en el conocimiento previo del niño y el punto de partida de su desarrollo y buscó explorar de manera lúdica la esfera socio-cognitiva. Para los alumnos, permitió experimentar las dificultades en la elaboración de recursos didácticos y, al mismo tiempo, puso de manifiesto la importancia del juego en la enseñanza de los niños. Consideraciones finales: La actividad propuesta era esencial para reflexionar sobre la importancia de las actividades lúdicas para el aprendizaje de los niños. La enfermera tiene el papel de educadora y a través de los juegos debe ayudar a los niños a cuidarse y promover la salud.

Palabras clave: Salud infantil; nutrición infantil; juegos y juguetes; Ensenãnza.

\section{Introdução}

Segundo Brasil (2018), a obesidade é considerada uma doença crônica, sob análise epidemiológica, como um dos mais graves problemas de saúde pública, a serem enfrentados nos países desenvolvidos e em desenvolvimento. É causada pelo aumento de tecido adiposo no organismo, que desencadeia fortemente diferentes riscos de morbimortalidade relacionados ás doenças cardiovasculares, osteomusculares, neoplásicas, diabetes, hipertensão arterial e acidente vascular encefálico (Moreira et al., 2013). As alterações no padrão alimentar ou uma alimentação disfuncional tem importante papel na determinação do elevado risco de desenvolvimento dessas doenças, que impactam diretamente na qualidade de vida do indivíduo nos diferentes ciclos de vida (Poll et al., 2020).

Nesse sentido, o consumo aumentado de alimentos processados e ricos em gorduras, a ingestão de alimentos industrializados, e a diminuição da atividade física caracterizam a mudança no padrão alimentar e o aumento da incidência da obesidade (Santos et al., 2019).

De acordo com o Brasil (2018), nos últimos treze anos, observou-se um aumento expressivo de obesidade 67,8\%, inicialmente 11,8\% em 2006 para 19,8\% em 2018. A maior taxa de crescimento foi entre adultos de 25 a 34 anos (84,2\%) e de 35 a 44 anos (81,1\%). Já em relação à obesidade infantil, o Ministério da Saúde e a Organização Panamericana da Saúde apontam que $12,9 \%$ das crianças brasileiras entre 5 e 9 anos de idade têm obesidade, assim como $7 \%$ dos adolescentes na faixa etária de 12 a 17 anos. Segundo estimativas, em 2025, 2,3 bilhões de adultos ao redor do mundo estarão acima do peso, totalizando 700 milhões de indivíduos com obesidade, ou seja, com um índice de massa corporal (IMC) acima de 30 (Brasil, 2018). 
O diagnóstico clínico da obesidade na infância e adolescência é realizado pelo índice de massa corpórea (IMC), preconizado por Quetelet em 1835, o qual é expresso através do peso em quilogramas dividido pelo quadrado da altura em metros (Peso/Estatura ${ }^{2}$ ). Esse índice é adotado como referência de medida para a obesidade pela Organização Mundial da Saúde (OMS) desde a década de 1997. Sendo assim, sobrepeso e obesidade são definidos como uma faixa de IMC de 25,0 - 29,9 Kg/m² e acima de 30,0 Kg/m², respectivamente. Ainda Segundo a OMS, a classificação da obesidade pode variar em: Obesidade grau I (IMC $>30$ e $<$ igual a 34,9 Kg/m²), Obesidade grau II (IMC > 35,0 e < igual 39,9 Kg/m²) e Obesidade grau III (IMC >40 Kg/m²) (Word health organization, 1995).

No Brasil, segundo os pesquisadores Aiello e colaboradores (2015) a prevalência de obesidade entre crianças e adolescentes identificadas em seu estudo de revisão sistemática com metanálise, corresponderam a um percentual de $14,1 \%$. Deste total, 6,5\% das crianças menores de 2 anos de idade apresentaram sobrepeso ou obesidade infantil. Os achados deste estudo ainda indicam necessária atenção e ações efetivas das Políticas Públicas de Saúde. Tais dados epidemiológicos são relevantes, uma vez que a chance de crianças acima do peso se tornarem adultos obesos é de $25 \%$ e a de adolescentes obesos, de $75 \%$, além dos diversos riscos à saúde física e mental, como já descritos anteriormente.

Diante disso, destaca-se que os primeiros anos de vida são essenciais para a incorporação de práticas alimentares saudáveis como medida efetiva para o controle e prevenção da obesidade infantil (Silveira, 2017). Sabe-se que a obesidade na infância e adolescência tende a continuar na fase adulta, se não controlada. É na infância que a formação destes hábitos sofre maior influência de fatores fisiológicos, ambientais e socioeconômicos, observados tanto no contexto familiar quanto na escola, levando ao aumento da morbimortalidade e diminuição da expectativa de vida (Silva et. al.,2016).

Desta forma, a detecção precoce de crianças com maior risco para o desenvolvimento de obesidade, juntamente com a tomada de medidas para controlar este problema, faz com que o prognóstico seja mais favorável á longo prazo (Silva et. al., 2016).

Somado a isso a família deve ser orientada sobre a prática da alimentação saudável, pois tem um papel decisivo na forma como a criança irá aprender a se alimentar, sobretudo pelas estratégias que os pais e cuidadores usam para estimular a alimentação. Há necessidade de refeições como: desjejum, lanches, almoço e jantar. O reconhecimento dos sinais de fome, saciedade e a compreensão acerca da capacidade de autocontrole da criança pequena em relação à ingestão alimentar contribuem para a formação de um comportamento alimentar adequado (Silva et al., 2016).

A escola também tem responsabilidade e deveres quanto à alimentação escolar. Ela deve adequar-se aos hábitos da região, evitando uso de alimentos não saudáveis (salgadinhos, refrigerantes, guloseimas). Deve-se ofertar diariamente para a criança frutas, verduras, legumes, fontes de calorias, minerais, vitaminas e fibras. O sal deve ser consumido com moderação, visando à formação de bons hábitos alimentares e prevenindo a hipertensão arterial (Silveira,2017).

Diante disso, é importante desenvolver métodos que promovam hábitos alimentares saudáveis de formas divertidas e lúdicas tornando esse momento agradável para as crianças. Sabe-se que durante a etapa escolar, as crianças de seis a onze anos começam a desenvolver capacidades de relacionamento entre eventos e representações mentais. Essa prática é denominada por Piaget como "Estágio de operações concretas". A criança utiliza-se de memórias e experiências prévias para auxiliar na interpretação do presente momento. Sendo capazes de separar e agrupar objetos de acordo com características comuns, colocando-as em uma ordem sensata e lógica (Pozas, 2020). Além disso, tendem a se ocupar com coleções de objetos, tais como, adesivos, cartas, carros e bonecas (Hockenberry et al., 2011). Por tanto utilizar ferramentas capazes de auxiliar nos hábitos alimentares podem contribuir para facilitar as representações mentais, criando situações de aprendizagem que podem despertar a criatividade dos alunos, proporcionando oportunidade de investigação e produção de conhecimentos. (Freemans et al., 2014; Sinnayah et al., 2019) 
Sendo assim, o brincar traz inúmeras vantagens para a formação do desenvolvimento cognitivo da criança, o ato de brincar ajuda a criança a desenvolver a diferenciação entre ação e significado, tirando conclusões concretas do ambiente em que habita (Vygotsky, 1998). Com isso, o lúdico torna uma proposta educacional no processo de ensino-aprendizagem, além de auxiliar no diagnóstico de personalidade das crianças (Rolim et al., 2008).

Ao brincar a criança expressa sua linguagem por meio de gestos e atitudes, sendo repleta de significados acrescidos de afetividade. Por isso a brincadeira deve ser encarada como algo sério e fundamental para o desenvolvimento, uma vez que compensa as pressões sofridas pelo cotidiano, por meio da capacidade de imaginar (Melo et al., 2005; Martins et al., 2016).

Em âmbito social, a escola e família constituem um dos mais importantes socializadores das crianças. Essas relações grupais promovem importantes interações sociais tendo como produto final o desenvolvimento da argumentação, o poder convencimento e negociação importantes para manter as amizades (Dessen et al., 2007).

Por isso, uma das formas mais interessantes de se brincar nessa faixa etária são os jogos em equipe. A brincadeira grupal ensina a criança a modificar ou negociar metas pessoais para atender às metas do grupo; ensina também que a divisão do trabalho é uma estratégia eficaz para se atingir um objetivo ou meta. As crianças aprendem a competir e a lidar com resultados não esperados (Hockenberry et al., 2011).

Dessa maneira, o objetivo do trabalho é relatar a experiência vivenciada em uma atividade lúdica proposta em um dos componentes curriculares obrigatório, disciplina "Assistência Integral à Saúde da Criança e do Adolescente I", no curso de Graduação em Enfermagem da Universidade Federal de Uberlândia, durante a criação e implementação do jogo "UNA os alimentos" como forma de ensinar sobre alimentação saudável para crianças de seis a onze anos de idade.

\section{Metodologia}

O presente artigo trata-se de um relato de experiência descritivo reflexivo elaborado a partir do referencial teórico (Pereira et al., 2018). Criado como proposta da disciplina Assistência Integral á Saúde da Criança e do Adolescente I, desenvolvido por discentes do sexto período do curso de graduação em Enfermagem da Universidade Federal de Uberlândia, no período de maio a junho de 2019.

A ideia inicial do jogo, foi inspirado no UNO original desenvolvido por Merlin Robbins e seus familiares (Gomes, 2013). A proposta apresentada pela docente era desenvolver um jogo para crianças na faixa etária de seis a onze anos. Assim, o grupo se reuniu para discutir sugestões de jogos para tal finalidade. Foram apresentados três jogos diferentes: Amarelinha, Jogo de argolas e o "UNA os alimentos". Após votação o grupo optou pelo o último e iniciou-se o processo de construção da arte das cartas, que foram desenvolvidas no programa CorelDraw ${ }^{\circledR}$. A temática das cartas foi fundamentada em fatores que influenciam a alimentação das crianças.

A escolha do tema foi baseada nas transformações que vem ocorrendo quando se trata de alimentação. Os produtos industrializados estão chegando mais facilmente à mesa das famílias, não só pela a praticidade do preparo mais também pelo baixo custo. Somado a esses fatores tem o sabor que é conferido a esses alimentos artificialmente e oferecem riscos a saúde, uma vez que esse tipo de alimento tem baixos valores nutricionais e muitos aditivos químicos. São ricos em açúcares e normalmente contém sódio acima do recomendado (Toloni et al., 2017). (Figura 1). 
Figura 1. Frente e verso das cartas do jogo; e como foram distribuídas as especificidades de cada grupo alimentar no verso da carta.

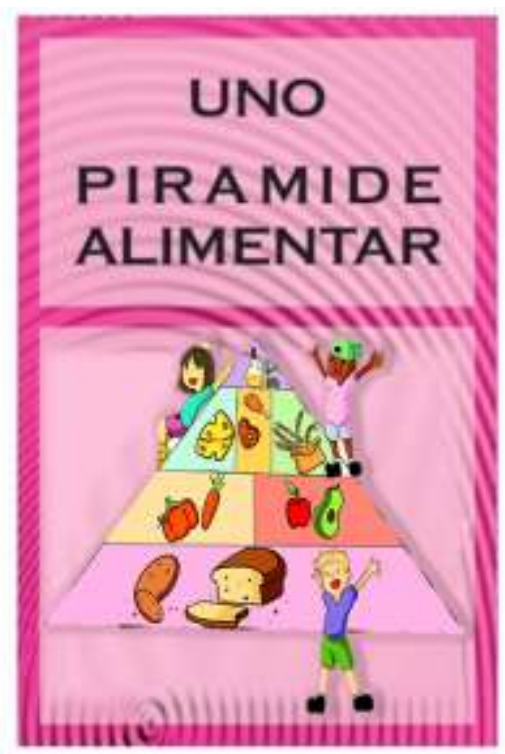

Frente da carta

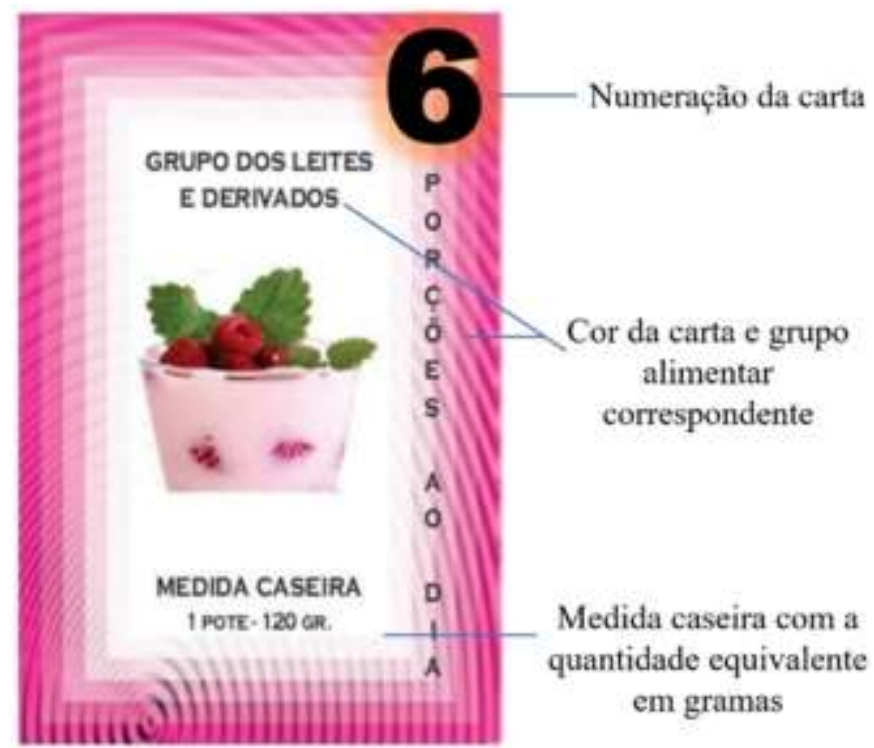

Verso da carta

Fonte: Maciel et al. (2020).

O jogo una os alimentos foi construído tendo como base a pirâmide alimentar e sua divisão por grupos alimentares proporcionando para toda a família compreensão acerca de uma alimentação diversificada (Philippi et al., 1999).

Dessa forma, a finalidade do jogo é facilitar a compreensão das crianças sobre a alimentação adequada, e a proposta se estende à família. Não há restrição de idade e deve promover aprendizagem sobre a temática com diversão, aliando o lúdico aos conceitos de uma dieta saudável e variada.

\section{Regras do jogo}

O baralho é composto por cartas de oito cores que correspondem a cada subgrupo alimentarem: verde (grupo das frutas), amarelo (açúcares), rosa (leite e derivados), laranja (feijão e leguminosas), marrom (carne e ovos), vermelho (óleos), roxo (legumes e verduras) e azul (grupo do arroz/cereal/massas/pães). As fileiras de cada cor variam entre 0 e 9. Existem três ações especiais para cada categoria de acordo com a cor da carta, identificadas como "pular", "comprar uma ou duas" e "inverter e mudar o grupo alimentar". As cartas regulares ou de ação são duplicadas no baralho. (Figura 2). 
Figura 2. Divisão das cartas do jogo por cores e grupos alimentares.

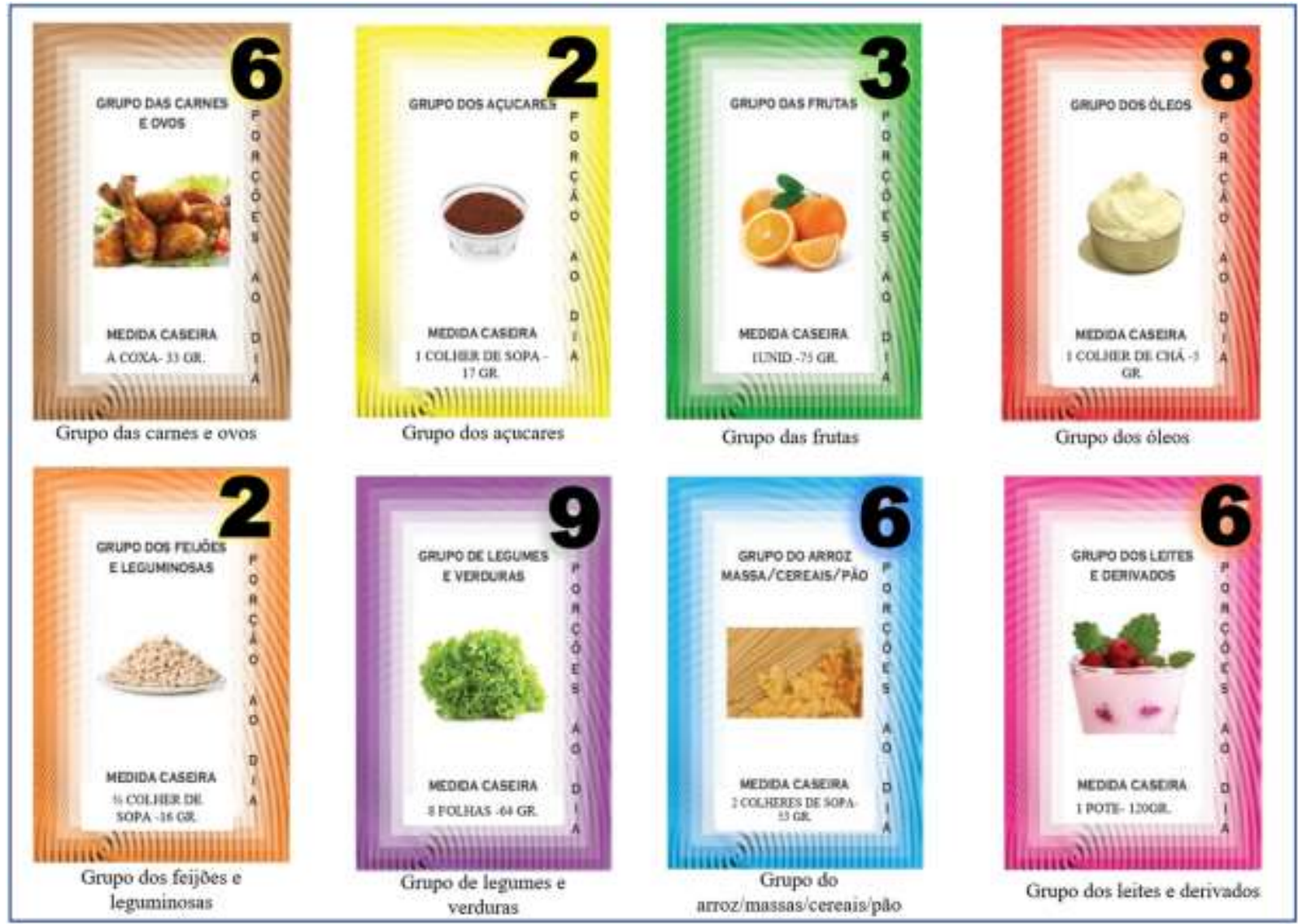

Fonte: Maciel et al. (2020).

O baralho é dividido em duas partes uma que será distribuída aos participantes e a outra que compões o "monte" (cartas que os jogadores podem adquirir durante o jogo).

Para começar a partida, são distribuídas cinco cartas a cada jogador, e uma será retirada do baralho para iniciar. Ao realizar a jogada, deve-se possuir um cartão do mesmo grupo alimentar ou com a mesma numeração daquele disposto ao centro; se não for compatível com as condições descritas o participante deve comprar uma no monte.

A nova carta adquirida deve atingir os requisitos caso ao contrário, a criança permanece com ela e passa a vez. É importante destacar que existem cartas com comandos especiais, e essas por sua vez, devem ser executadas durante a partida. Se as cartas do monte esgotarem, as que foram descartadas devem ser embaralhadas e utilizadas novamente. Ganhará o jogador que não tiver cartas na mão.

\section{Aplicação em sala}

O jogo "UNA os alimentos" foi executado em sala de aula com os graduandos do sexto período do curso de Enfermagem. Participaram 30 estudantes resultando em 3 grupos de 10 integrantes, o tempo destinado ao Una os alimentos foi de 15 minutos com rodadas que duraram em média de 5 á 15 minutos. Para melhor disposição do ambiente as mesas foram organizadas em círculo tendo em cada grupo um mediador cuja função era observar se a execução estava sendo correta, bem como a interação da equipe. Contou-se ainda, com outros quatro discentes designados para apresentar o jogo e explicarem as regras. As normas foram apresentadas a todos os jogadores, em seguida realizamos a orientação das funções de cada carta e os 
grupos alimentares, a importância deles, como pode ser substituída por outros alimentos do mesmo grupo alimentar e ainda assim manter uma alimentação saudável, gostosa e nutritiva.

No decorrer do jogo verificou-se que esse era eficiente mesmo com alunos da graduação, os participantes estavam empenhados e se divertiram bastante. Todas as equipes tiveram um ganhador, premiados ao final da atividade.

Recebemos um feedback positivo, a atividade despertou a curiosidade dos participantes e todos tiveram facilidade na identificação dos grupos alimentares.

Vale ressaltar que o jogo pode ser utilizado em qualquer ambiente, sem a necessidade de muitos recursos. Ao encerramento da ação verificamos a viabilidade do jogo com crianças de 06 a 11 anos de idade. Os discentes sugeriram como melhoria a modificação da qualidade das cartas, uma vez que, foram impressas em papel couché e as crianças poderiam danificálas durante o manuseio.

\section{Aplicação com as crianças}

Foi realizado um jogo piloto com crianças de 6 á 11 anos no âmbito familiar dos discentes, o grupo foi composto por 7 integrantes distribuídos na faixa etária citada acima. Durante a brincadeira notou-se que as crianças com idade inferior a sete anos apresentaram dificuldades no reconhecimento das cartas, necessitando de um mediador para realizar a leitura e explicar os grupos alimentares. Nesse contexto, o tempo de atividade foi de 2 horas, resultando em diversão; após algumas partidas foi observada uma assimilação do conteúdo presente nas cartas, pelas crianças. Evidencia-se que apesar de algumas dificuldades o jogo atingiu o objetivo de reconhecimento dos grupos alimentares a suas porções.

\section{Discussão}

A educação em saúde é fundamental para auxiliar em todas as mudanças e transformações da infância, pois auxilia a estabelecer hábitos saudáveis e contribui para a construção do processo educacional.

$\mathrm{Na}$ fase dos seis aos onze anos, o ritmo de crescimento é constante, sendo próximo ao estirão da adolescência que é uma fase de acentuado ganho de peso, altura e puberdade. É também uma fase em que a criança adquire maior independência e socialização. Nesse sentido, a educação voltada à alimentação é fundamental para a manutenção da saúde e evitar o adoecimento precoce (BRASIL, 2012).

O jogo foi planejado de maneira a considerar o saber anterior da criança e o ponto de partida do seu desenvolvimento. Explorou-se de forma lúdica o âmbito sócio cognitivo da criança, bem como promover o conhecimento acerca dos grupos alimentícios, visando à compreensão da necessidade de uma alimentação balanceada.

As crianças já possuem um conhecimento mesmo que superficial sobre os alimentos, o que observamos na aplicação do jogo é que com a atividade elas conseguiam organizar melhor os alimentos dentro do seu grupo alimentar. Porem o jogo é mais efetivo se houver um mediador para ajudar a transmitir as informações contidas nas cartas sendo esse a principal limitação encontrada na aplicabilidade do "Una os alimentos". Constatamos que na medida em que foram realizadas rodadas do jogo apareceram alguns problemas estruturais, como por exemplo, como ficaram organizadas as informações na carta que precisa ser lida para atingir o objetivo. Durante a leitura das cartas pelas crianças cada uma começava de um a informação diferente isso precisa ser definido nas regras do jogo, a ordem de leitura das informações das cartas. Outro esclarecimento que precisa ser acrescentado nas regras do jogo é a de que o jogador precisa proceder à leitura em voz alta para que os jogadores se familiarizem com os grupos alimentares e suas porções diárias.

$\mathrm{Na}$ execução em si dos comandos que o jogo necessita das crianças foram identificadas pequenas dificuldade, no que tange o reconhecimento das cartas e a leitura rápida; esses pontos foram facilmente contornados com a presença do mediador e 
pela familiaridade ao jogo, adquiridos durante as partidas. Além disso, o tipo de letra utilizado nas cartas favorece a leitura de crianças em idade de alfabetização que costumam iniciar a leitura por letras em bastão.

Os jogadores mostraram interesse pela atividade e ao final dos jogos podemos observar que os alimentos que são mais consumidos na sua rotina alimentar já eram identificados pelo grupo alimentar, por exemplo, arroz, batata, e o macarrão como carboidratos; feijão, carne como proteínas.

Estudos recentes demostram que crianças têm preferências por determinados grupos alimentares e são mais seletivas quando se trata da categoria: legumes/verduras e grãos/cereais. Nesse cenário, os jogos favorecem uma mudança nos costumes alimentares, sendo uma ferramenta capaz de viabilizar o contato da criança com os alimentos; reforçando o consumo saudável e aceitação de variedade de alimentos na rotina (PANOSSO et al., 2018).

No geral, o jogo atingiu o objetivo de passar informações sobre alimentação saudável de forma lúdica. No entanto, por se tratar de um projeto piloto, as cartas e as regras precisam ser melhoradas. Buscar parcerias com o curso de nutrição foram umas das estratégias propostas pelos integrardes do grupo para aperfeiçoar a qualidade das informações contidas nas cartas.

Ao final do jogo as crianças tiveram um bom aproveitamento das informações, foram capazes de diferenciar os grupos de alimentos, porções ideais e as substituições deles dentro das categorias. Um ponto a ser destacado é que o jogo seria mais enriquecedor se envolvesse a equipe multiprofissional de forma contínua nos diferentes espaços, notadamente a equipe de nutrição que possui mais conhecimento a cerca da temática; somada a isso, é de suma importância o envolvimento dos familiares e cuidadores para que haja um campo de prática dos conhecimentos observados.

A elaboração do jogo proporcionou aos acadêmicos vivenciar as dificuldades no desenvolvimento de recursos didáticos e ao mesmo tempo evidenciou a importância do lúdico no ensino de crianças. Mediante a esses entraves, a equipe de enfermagem pode adotar o jogo como uma tecnologia de ensino nos espaços de educação e saúde, ou seja, aplicação nas escolas, nas salas de esperas das unidades básicas de saúde e até mesmo disponibilização de materiais para realizar as brincadeiras no ambiente familiar.

\section{Considerações Finais}

A prevalência de obesidade em crianças e adolescentes no Brasil é elevada e necessita urgentemente de medidas para prevenir as consequências associadas à doença, bem como medidas para reduzir o impacto, em curto prazo, do sobrepeso e da obesidade infantil. Por tanto jogos que abordam a temática alimentação destinados ás crianças podem ser importantes para a construção de hábitos alimentares saudáveis. Esse é o objetivo do jogo relatado "UNA os alimentos".

A proposta de elaboração do jogo foi fundamental para proporcionar reflexões de como atividades lúdicas podem ser importantes para o aprendizado das crianças, além de auxiliar no desenvolvimento cognitivo e motor. Através do brincar, ele ensina a criança experimentar o mundo, as possibilidades na construção das relações sociais, a mesma elabora sua autonomia de ação e organiza as emoções. Além disso, o jogo proporciona a criança compreensão do mundo à sua volta, aprende regras e testa habilidades. Por tanto, o profissional da saúde deve proporcionar a comunidade informações e recursos que demostram aos familiares a importância do brincar para o desenvolvimento da criança, pois é brincando que se aprende. Vale acrescentar que o tema trabalhado é vasto e passível de uma pesquisa mais detalhada, na qual os jogos poderão ser utilizados como instrumento na etapa de intervenção, sendo possível fazer o comparativo dos hábitos iniciais e dos hábitos posteriores a ação. Essas atitudes podem contribuir para melhoria da qualidade da alimentação, e colocar as crianças como sujeito ativo no processo de promoção e prevenção á saúde. 


\section{Referências}

Andrade, B. H. D. (2019). Evolução da cobertura e do monitoramento e estado nutricional de individuos cadastrados no sistema de vigilancia alimentar e nutricional: de 2008 a 2018. World Health Organization. (1998). Obesity: preventing and managing the global epidemic: report of a WHO consultation on obesity, Geneva, 3-5 June 1997 (No. WHO/NUT/NCD/98.1). World Health Organization.

Brasil, I. B. G. E. (2010). Instituto Brasileiro de geografia e Estatística. Censo demográfico, 2010.

Brasil, V. (2018). Vigilância de fatores de risco e proteção para doenças crônicas por inquérito telefônico. Brasília: Ministério da Saúde, 132.

Cavicchia, D. D. C. (2010). O desenvolvimento da criança nos primeiros anos de vida. IN Caderno de Formação: Formação de Professores Educação InfantilPrincipios e Fundamentos, 1, 13-27.

da Cruz Rodrigues, L. M., Rodrigues, R. S., de Araújo, S. A., Mendes-Rodrigues, C., Figueiredo, V. N., \& da Silva, P. C. D. S. (2021). Avaliação da satisfação quanto ao jogo educativo NeuroGame-Card como estratégia de ensino em Enfermagem. Research, Society and Development, 10(7), e14510716368e14510716368.

da Cruz Santos, J., de Carvalho, D. M. A., \& de Pinho, L. (2019). Consumo de alimentos ultraprocessados por adolescentes. Adolescencia e Saude, 16(2), 5663.

de Aguiar Toloni, M. H., Longo-Silva, G., Alvarenga, A. P., Konstantyner, T., \& Taddei, J. A. D. A. C. (2017). Preditores da introdução de alimentos industrializados na infância: estudo em creches públicas. O Mundo da Saúde, 41(4), 644-651.

Dessen, M. A., \& Polonia, A. D. C. (2007). A família e a escola como contextos de desenvolvimento humano. Paidéia (Ribeirão Preto), 17, 21 -32.

Freeman, S., Eddy, S. L., McDonough, M., Smith, M. K., Okoroafor, N., Jordt, H., \& Wenderoth, M. P. (2014). Active learning increases student performance in science, engineering, and mathematics. Proceedings of the national academy of sciences, 111(23), 8410-8415.

Hockenberry, M. (2011). Wong's Fundamentos Enfermagem Pediátrica. Elsevier Health Sciences.

Maria Aiello, A., Marques de Mello, L., Souza Nunes, M., Soares da Silva, A., \& Nunes, A. (2015). Prevalence of obesity in children and adolescents in Brazil: a meta-analysis of cross-sectional studies. Current pediatric reviews, 11(1), 36-42.

Melo, L. D. L., \& Valle, E. R. M. D. (2005). O brinquedo e o brincar no desenvolvimento infantil. Psicol. argum, $43-48$.

Mesquita, D. K. M., Maia, E. C., França Junior, D. B., Santos, H. F. D. A. C., Costa, M. H. S. N., Campos, C. S. D. S., ... \& Pessoa, F. S. (2014). Saúde da criança: crescimento.

Moreira, N. F., Muraro, A. P., Brito, F. D. S. B., Gonçalves-Silva, R. M. V., Sichieri, R., \& Ferreira, M. G. (2013). Obesidade: principal fator de risco para hipertensão arterial sistêmica em adolescentes brasileiros participantes de um estudo de coorte. Arquivos Brasileiros de Endocrinologia \& Metabologia, 57, 520526.

Palombo, C. N. T., Duarte, L. S., Fujimori, E., \& Toriyama, Á. T. M. (2014). Uso e preenchimento da caderneta de saúde da criança com foco no crescimento e desenvolvimento. Revista da Escola de Enfermagem da USP, 48, 59-66.

Panosso, M. G., Gris, G., \& Souza, S. R. D. (2018). Efectos de un juego de mesa sobre la selección e ingesta de alimentos de los niños. Revista Psicologia e Saúde, 10(2), 103-123.

Philippi, S. T., Latterza, A. R., Cruz, A. T. R., \& Ribeiro, L. C. (1999). Pirâmide alimentar adaptada: guia para escolha dos alimentos. Revista de nutrição, 12, $65-80$.

Poll, F. A., Miraglia, F., D'avila, H. F., Reuter, C. P., \& Mello, E. D. (2020). Impacto da intervenção no estado nutricional, consumo de processados e qualidade de vida de adolescentes com excesso de peso々̌ Jornal de Pediatria, 96, 621-629.

Pozas, D. (2020). Criança que brinca mais aprende mais: a importância da atividade lúdica para o desenvolvimento cognitivo infantil. Editora Senac Rio.

Rodrigues, R. S., Rodrigues, L. M. D. C., Mendes-Rodrigues, C., \& Figueiredo, V. N. (2020). Elaboração de jogo de tabuleiro educativo para avaliação dos pares de nervos cranianos-"Neurogame-card"

Rolim, A. A. M., Guerra, S. S. F., \& Tassigny, M. M. (2008). Uma leitura de Vygotsky sobre o brincar na aprendizagem e no desenvolvimento infantil. Revista Humanidades, 23(2), 176-180.

Silva, G. A., Costa, K. A., \& Giugliani, E. R. (2016). Alimentação infantil: além dos aspectos nutricionais ł̧. Jornal de Pediatria, 92 , 2-7.

Silveira, M. D. G. G. (2017). Prevenção da obesidade e de doenças do adulto na infância. Editora Vozes Limitada.

Silveira, M. D. G. M., \& Gentil, V. K. (2016). Relato de experiências com lúdico na sala de aula. CCNExt-Revista de Extensão, 3, $1239-1243$.

Sinnayah, P., Rathner, J. A., Loton, D., Klein, R., \& Hartley, P. (2019). A combination of active learning strategies improves student academic outcomes in firstyear paramedic bioscience. Advances in physiology education, 43(2), 233-240.

Status, W. P. (1995). The use and interpretation of anthropometry: report of a World Health Organization (WHO) expert committee. Geneva, Switzerland: World Health Organization.

Tassara, V., Norton, R. C., \& Marques, W. E. U. (2010). Importância do contexto sociofamiliar na abordagem de crianças obesas. Revista Paulista de Pediatria, 28(3), 309-314. 
Research, Society and Development, v. 10, n. 9, e2710917820, 2021

(CC BY 4.0) | ISSN 2525-3409 | DOI: http://dx.doi.org/10.33448/rsd-v10i9.17820

Vygotsky, L. (1984). Semenovich. A formação social da mente. Tradução: José Cipolla Neto, Luís Silveira Menna Barreto, Solange Castro Afeche. São Paulo: Martins Fontes.

Pereira, A. S., Shitsuka, D. M., Parreira, F. J., \& Shitsuka, R. (2018). Metodologia da pesquisa científica.[e-book]. Santa Maria. Ed (pp. 3-9). UAB/NTE/UFSM. Disponível em: https://repositorio. ufsm. br/bitstream/handle/1/15824/Lic_Computacao_Metodologia-Pesquisa-Cientifica. pdf. 